\title{
Economic Significance of Digital Libraries: A Structured Review
}

\author{
Azino O. Akpokurerie \\ Adeyemi College of Education Ondo, \\ Ondo State \\ azinoakpos@gmail.com
}

\author{
Marian Nina- Okpousung \\ Department of library and information science \\ Delta State Polytechnic \\ Ogwashi-uku, \\ monluv2002@yahoo.com
}

\begin{abstract}
Traditional concept of library is mostly based on print materials; a very important part is how the introduction of technology has affected the communication and interaction between librarians and library users. In traditional libraries users have face-to-face contact with the librarians. They communicate through their eyes; they use oral or nonverbal communication such as facial expression. Presently, attention is shifting from print towards materials in electronic form. Digitalization has increased the means to make materials more readily available by adding advanced search features to access these documents.. Digital mode gives better transmission speed and quality than analog transmission. This paper defined digital libraries, their roles and advantages, their ability to meet users and library economic needs. Challenges related to inadequate finance, infrastructure and lack of expertise are among issues hindering digital libraries establishment. Recommendations made were that specific legislation and financial support be given to libraries from all tiers of government as well as consultation with right owners and other stakeholders is essential to know their peculiarity and need in establishing digital library.
\end{abstract}

Keywords: digital library, library users, electronic resources, digitalization

\section{Introduction}

Digital library allows information materials to be stored in computer system in a form that can be manipulated and delivered in ways that the conventional version of the material cannot be stored. The primary benefit of these digital projects is the return to patrons from accessing these materials. Once digitized, stored, and made accessible over a network or the Internet, the materials are more easily accessible to patrons. Patrons who previously had to travel to a library to access hard copies of tmaterials can now view them online from home or the office. Libraries are established to meet information needs of the society, therefore, they play a vital role in the educational and societal development by providing relevant and useful information to the people. Today, a lot of information is being generated 
through print as well as other medium. Traditional library is mostly based on print, currently; there is a change towards electronic form. Digitalization has increased the means to making information as well as materials carrying this information more readily available by adding advanced search features to access the document. We are living in an information society and ICT is an increasing phenomenon.

During the last decade, the use of technology has become an essential part of the institutional objective of libraries.This study seeks to explore relevant literature in determining:

1. digital libraries and their roles in relation to current economic needs?

2. Significance of digital library?

3. challenges and problems of digital libraries in Nigeria?

\section{What is a digital library?}

We must recognize that there are many views as to what digital library means. Is it an electronic library? A digitized collection? Scientific repository of software and models? A publisher's collection? Or the world wide web itself? Kothart (2008) defined digital library as electronic information collection containing large and diverse repositories of digital object, which can be accessed by a large number of geographically distributed users. Digital library are set of electronic resources and associated technical capabilities for creating, searching and using information. Digital library may not necessarily be network but would largely contain digitized information along with print based publications. They could be referred to as computerized network system in which all information is stored in electronic format which can be access and transmitted through network enabling retrieval of needed information by a large number of users. These libraries will give the citizen online access to books, to local historical records, to archive films, and museum objects - and provide services so they can use them. Digital libraries cannot function in isolation when the process is not set. No wonder, Akintunde (2007) observed that digitization is the process of translating a piece of information such as sound recording, picture or video into bits in his opinion,

With the view of satisfying economics needs, digital library guides making of choices made by individuals, institutions and communities with regard to alternative uses of scarce resources to satisfy the desire for digital content and digital libraries. Traditional print publishers have 
found that additional costs of production are necessary to publish a journal in both print and digital format, increasing subscription costs to libraries that require access to both forms.

Digital costs are lumpy, with a large fixed cost of production, and zero marginal cost to produce an additional digital copy over the Internet. The role of the library as intermediary is critical in the pricing of information. Libraries purchase information materials and provide access to patrons typically without an access fee. Patrons efficiently use the information since, in the networked digital environment, providing the information has no marginal costs and patrons are not charged for access. The charge to libraries covers the cost of production of the information while the absence of a charge for patrons insures economic efficiency.

\section{What are the roles of digital library?}

1. The conventional roles of a library are recognized as knowledge archival, safeguarding and maintaining the culture, knowledge dissemination, knowledge sharing, information retrieval, education, and social building contacts. Neal (1997) Opined that digital library is a library in which most of the information is available in electronic form, allowing users to access many of the library's services remotely via the internet, move hard-copy content to digital format to help improve access

2. Digital library offer better opportunity for users to deposit as well as use information. Thus, students and teachers can simply be publishers as well as readers in digital libraries systems. Marchionini and Maurer, (1995). Covi and Cragin (2004) emphasized that students and faculties require and prefer access to electronic sources delivery systems and to networked information. This is a clear sign that in order to meet the information needs of the academic community, academic libraries must provide online information services.

3. Digital library can disseminate information across a network and the users can retrieve information in the same ways.

4. Digital library are global virtual libraries i.e. the library of thousands of networked electronic libraries. 
5. Digital library need preserve print historical documents/information materials for the foreseeable future.

\section{Advantages of digital libraries}

1. Ubiquity is one clear edge: a single electronic copy can be accessed from a great many locations, and to many simultaneous users. Copies can be delivered with electronic speed, and it may be possible to reformat the material to the convenience of the reader (e.g. in larger type size for those with limited sight, or in order to fit a smaller screen). Since readers get a screen display of the object, rather than carrying away the physical object, loss rates by theft may be much reduced some CD-ROM systems, however, are vulnerable to theft of this sort. Unfortunately, although the library may be immune from losing access to its own copy via theft. In the digital world, there is a different kind of theft in which the copyright holder loses control, rather than the library losing its copy.
2. Another important role is preservation. Digital information can be copied without error. As a result, preservation in a digital world does not depend on having a permanent object and keeping it under guard, but on the ability to make multiple copies, assuming that at least one will survive. The major risk to digital objects is usually not physical deterioration but technological obsolescence of the devices to read them.

3. Digital storage also permits libraries to expand the range of material they can provide to their users. For example, both audio cassette tapes and vinyl records pose problems to libraries; neither will stand a large number of playing without deterioration

4. Digital material can also permit access to fragile photographs, video tapes, and to the new kinds of multimedia materials that are created only on computers and simply have no equivalent in any traditional format. 
5. Digital libraries provide better retrieval and faster communication

6. User from many locations can access simple electronic information, can copy, print and preserve at his location or wherever he desires.

7. Information search is easy

\section{Economic Significance of Digital Library}

Libraries' social and economic benefits are, by definition, intangible. As such, they become diluted on receipt by wider society, government and organisations. They provide a way of preserving social or organisational knowledge and of making it available to members. However, it is the latter, through their activities, which extract value by applying the knowledge acquired from the information stored, catalogued and processed in these centres. It is not possible to calculate their economic value using conventional market-based supply and demand criteria. Therefore, the challenge is to create a methodology capable of estimating that social and economic contribution to society as a whole and, at the same time, of measuring the return on investment. Digital libraries are needed both to let libraries offer new and improved services, and to deal with a cost crisis now affecting their current services. Dreams of computer-based libraries, which have been around for decades, are now practical. There are libraries which already spend more than half their acquisitions budget on electronic resources, rather than paper. Meanwhile, many university libraries are finding it impossible to maintain their traditional collecting practices within the constraints of their institutional budgets. They look eagerly to the new technology as a solution. The current scholarly publishing system is breaking down. Libraries can afford an ever decreasing fraction of the world's publications. Increases in journal and monograph prices, currency shifts, and increases in the number of publications are all making it harder for libraries to afford their previous level of purchases. Nearly every university is reviewing its subscriptions and canceling journals. The whole system is near collapse.

Since much of the cost of printing many scholarly journals is expensive, a loss of subscriptions means that the cost to the remaining subscribers must be increased. This will cause still more price increases and 
then cause additional libraries to stop purchasing those journals. The result has been a globally strange optimization, despite the large economy of scale in printing journals, libraries work out sharing arrangements because they cannot afford to buy more copies. This is counter-productive, since it leads to still fewer subscriptions and still higher prices, but there is nothing one library can do about the situation. It is a problem independent of technology; exactly the same situation is observed with CDROMs, with libraries working out ways to provide network access, when CD-ROMs again are a technology with huge economies of scale and low incremental costs but produced for libraries in small runs.

In addition to problems buying material, libraries are beset with increasing costs for buildings and storage, and with a steadily growing problem of acid paper books which are deteriorating and need treatment. Something over 100 million books in the United States are brittle, and there is as yet no suitable solution (photocopying and deacidification are both too expensive, and microfilm is disliked by many users). Building costs also increase steadily, and on many campuses there is simply no room to put another large library even if the funds are available to build it. Since most universities do not monetize space, this rarely appears in a library budget, but the cost of space is probably comparable to the cost of materials in some realistic accounting.

\section{Challenges of Digital Library in Nigeria}

In this digital era, librarians are facing many challenges to redefine their role and also to redesign the provision of information services. The digital library involves a huge investment for digitalization of their existing collection and acquisition of digital resources. Pomerantz and Marchionini, (2007) considered the following as the challenges being faced by libraries as well as librarians.
a) Intellectual property rights challenges.
b) Technological challenges.
c) Challenges related to knowledge and resource management.
d) Service oriented challenges.
e) Challenges related to preservation.
f) Challenges related to inadequate finance and infrastructure.
g) Lack of expertise.
h) Security challenges. 
In digital era, librarians are expected to play a vital role to suit the changing needs of tomorrow's information community. Traditionally, librarians have often organized and classified information in such a way that they can find the information on behalf of the users. In the other words, librarian has fulfilled physical role of adviser, interpreter and mediator for user's information needs. He should embrace the entire new information cycle form creation; restructuring and representation of information to its dissemination and use. The librarian has to gear up to face the demand and challenges and this can be achieved only through fundamental rethinking and radical redesigning of library processes to achieve dramatic improvements in critical contemporary measures of performance of librarian.

\section{Recommendations}

Having considered the important of digital libraries and their economic impact on libraries and its users, the following are recommended.

1. Specific legislation and financial support from national, state and local governments is required to bridge the digital divide and to ensure sustainable access. Any long-term strategy must aim to bridge the digital divide and to strengthen the development of education, literacy, culture - and most of all - to provide access to information.

2. All tiers of government should encourage libraries to collaborate with other cultural and scientific heritage institutions to provide rich and diverse digital resources that support education and research, tourism and the creative industries.

3. Consultation with rights owners and other stakeholders is essential. Designers and implementers of digital libraries should consult fully with indigenous communities,

\section{Conclusion}

The digital libraries are the ultimate beauty and rhythm of information flow. That is the dream of information providers and seekers in the knowledge center system in a distributed network environment. The digital libraries satisfy the information needs of users in all possible ways. Digital libraries offer a wide range of new access' opportunities that are absent in the traditional environment, including remote access, 24-hour access and multiple users for single sources. The arrival and 
proliferation of electronic resources and digital libraries have already influenced and changed the ways academics and scholars use print resources and traditional libraries. It has also sparked a new wave of literature on the perceptions and preferences of print and electronic resources. Reason behind so many libraries and other institutions, turned their hands to creating digital libraries is to do with advances in information technology - increasingly both academic and recreational material is being made available in electronic formats, either in addition to or instead of print. The costs of creating, storing and disseminating electronic information have decreased, and the technology to support distribution and access is widespread. Rising acquisition and subscription fees have forced libraries to seek other ways to make information available and content aggregators and ebook publishers are providing the means. Most significantly, digital libraries support service improvement. Information search and navigation across electronic information resources is much faster, with enriched points of access, and alternative methods for browsing and exploration. The resources themselves can be segmented, rearranged, annotated and enhanced in ways not possible before, and can be directly integrated with desktop productivity tools for local analysis and processing. A digital environment enables cross-community interactivity and collaboration, regardless of physical location Stewart, C (2012). Also, digitalization presents opportunities for long-term preservation of bodies of knowledge, if not of the original carriers of that knowledge. It is also crystal clear that libraries are required to perform all the knowledge management tasks in the digital era. Librarians will have to equip themselves for capabilities to link with global trends for the ultimate benefits of formation seekers. Librarians recognize their role in much more than custodians of knowledge. 


\section{References}

Stephen A. Akintunde \& Ruth Anjo (2012.) Digitizing Resources in Nigeria: An Overview. Retrieved3/7/2017 from http://www.netlibrarynigeria.net/downloads/ Akintunde.doc1.pdf.

Covi, L. \& Cragin, M. (2004). Reconfiguring control in library collection development: A conceptual framework for assessing the shift toward electronic collections. Journal of the American Society for Information Science, 55(4), 312-325.

Kothart, D.V (2008) Digital library: in Information literacy in the digital age. New Delhi. Ess publisher.

Marchionini, G., \& Maurer, H. (1995). The roles of digital libraries in teaching and learning. Communications of the ACM, 38(4), 67-75.

Neal, S. (1997). The virtual library-a market perspective. Asian Libraries, 6(1/2), 57-66.

Pomerantz, J. \& Marchionini, G., (2007).

The digital library as place. Journal of Documentation, 63(4), pp.505-533.
Stewart,T. (1997), Intellectual capital: The new wealth of organizations. London, Brealey,

Stewart, C., 2012. Preservation and access in an age of E-science and electronic records: Sharing the problem and discovering common solutions. Journal of Library Administration, 52(3), pp.265- 278. 